\title{
ESCOLARIZACIÓN DE LA EDUCACIÓN PARVULARIA EN CHILE: CONSENSOS ENTRE ACTORES DEL CAMPO SOBRE SU DEFINICIÓN, CAUSAS Y PROPOSICIONES ${ }^{1}$
}

\author{
Marcela Pardo², María José Opazo ${ }^{3}$, Pablo Rupin ${ }^{4}$
}

\section{RESUMEN}

El artículo presenta los resultados de una investigación cuyo objetivo fue identificar los principales consensos entre actores del campo acerca de la definición, causas y proposiciones de la escolarización de la educación parvularia en Chile. Basado en un diseño cualitativo, de nivel exploratorio, la recolección de datos se realizó mediante entrevistas individuales a autoridades y exautoridades nacionales del nivel, y a directoras, líderes pedagógicos del nivel y educadoras de párvulos $(\mathrm{N}=43)$ de cinco jardines infantiles y cinco escuelas financiadas con recursos públicos, que imparten Nivel Medio y/o Nivel Transición, seleccionados por conveniencia. Habiendo sometido los datos a análisis de contenido y luego a triangulación, los resultados obtenidos muestran que los tres grupos de actores definen la escolarización como un fenómeno de orden pedagógico que afecta principalmente al Nivel Transición, valorándolo negativamente e identificando como causas un conjunto de elementos culturales y factores estructurales de la calidad del nivel. Dichos actores propusieron medidas de política pública para revertirla.

Conceptos clave: educación parvularia, escolarización, preparación para la escuela, pedagogía infantil

\section{SCHOOLIFICATION OF EARLY CHILDHOOD EDUCATION IN CHILE: AGREEMENTS AMONG ACTORS IN THE FIELD ON ITS DEFINITION, CAUSES AND PROPOSALS}

\footnotetext{
ABSTRACT

This article presents findings from a research aimed at identifying the main consensus on the definition, causes and proposals on the schoolification of early childhood education in Chile, according to the vision of actors in the field. Relying on an exploratory and qualitative design, data was gathered through individual interviews to both current and former national governmental authorities, as well as to principals, pedagogical leaders and early childhood teachers $(\mathrm{N}=43)$ from 5 early childhood centers and 5 schools, selected for convenience, all of

1 Proyecto apoyado financieramente por CNED/Convocatoria 2019, y por el Proyecto Basal FB0003 del Programa de Investigación Asociativa de CONICYT.

2 Universidad de Chile, Santiago, Chile. Contacto: marcelapardo@ciae.uchile.cl

3 Universidad de Chile, Santiago, Chile. Contacto: mariajose.opazo@ciae.uchile.cl

4 Universidad de O'Higgins, Rancagua, Chile. Contacto: pablo.rupin@uoh.cl
} 
144 ESCOLARIZACIÓN DE LA EDUCACIÓN PARVULARIA EN CHILE: CONSENSOS ENTRE ACTORES DEL CAMPO SOBRE SU DEFINICIÓN, CAUSAS Y PROPOSICIONES - M. Pardo, M. Opazo, P. Rupin

them publicly funded, and encompassing classrooms for, 2-, 3-, 4- and 5-year-olds' classrooms. Having conducted content analysis and data triangulation, findings show that these three groups of actors defined the schoolification of early childhood education as a pedagogical phenomenon, which affects mostly 4- and 5-year -olds' classrooms, valuing it negatively. Identifying as causes a number of cultural elements and structural factors of quality, they proposed measures of public policy to revert this trend.

Key concepts: early childhood education, schoolification, school readiness, early childhood pedagogy 


\section{Introducción}

La escolarización de la educación parvularia ${ }^{5}$ emergió hace casi dos décadas como materia de preocupación dentro del ámbito de la política pública. Definida como la adopción de contenidos y métodos pedagógicos tradicionales en el sistema escolar, este fenómeno ha sido caracterizado en términos de la conformación de grupos de gran tamaño en el aula, la priorización del aula como espacio para el aprendizaje (relegando los espacios abiertos), el fomento de las interacciones asimétricas o unidireccionales entre la educadora y los niños ${ }^{6}$ (reduciendo aquéllas entre los niños mismos), la reducción de las oportunidades de juego (limitándolo al tiempo del recreo) y la promoción de la disciplina (restringiendo el diálogo y el movimiento espontáneo). Así, también, la escolarización de la educación parvularia ha sido descrita por un fuerte énfasis en la lectura, la escritura y las matemáticas (en desmedro de la promoción de habilidades socioemocionales, artísticas y motoras de los niños) (Moss, 2012; OECD, 2006). Esta tendencia ha sido ilustrada con la idea de que la educación básica ha "colonizado" a la educación parvularia (OECD, 2006, p. 62), subsumiéndola como parte suya $y$, en consecuencia, llevándola a perder sus tradiciones pedagógicas distintivas (OMEP, 2010) ${ }^{7}$.

Principalmente observada en grupos de niños de cuatro y cinco años, la escolarización de la educación parvularia ha cobrado mayor fuerza en aquellos países que han intentado facilitar las transiciones de los niños entre ambos niveles educativos (Cameron \& Moss, 2011; OECD, 2012) o preparar a los niños para su ingreso a la educación básica (Pianta, 2007, p. 6), pasando a entender este nivel educacional como "preprimario" o "preescolar". Esta tendencia ha sido reportada en países diversos, incluyendo, por ejemplo, Inglaterra (Moss, 2002),

5 En Chile se denomina "educación parvularia" al primer nivel del sistema educacional nacional. Éste atiende integralmente a los niños desde su nacimiento hasta el ingreso a la educación básica. En la literatura internacional se denomina usualmente "educación temprana", "educación inicial" o "educación infantil".

6 Este artículo adhiere a la perspectiva de género; sin embargo, con el solo propósito de facilitar la lectura, utiliza el género masculino para referirse a niñas y niños.

7 Cabe hacer notar aquí una precisión relevante: en este artículo el concepto de "escolarización" no se refiere al ingreso de los niños a la institución escolar. Éste, de hecho, puede darse bajo enfoques escolarizados o no. 
146 ESCOLARIZACIÓN DE LA EDUCACIÓN PARVULARIA EN CHILE: CONSENSOS ENTRE ACTORES DEL CAMPO SOBRE SU DEFINICIÓN, CAUSAS Y PROPOSICIONES - M. Pardo, M. Opazo, P. Rupin

Nueva Zelanda (Alcock \& Haggerty, 2013), Islandia (Gunnarsdottir, 2014), Dinamarca (Brogaard Clausen, 2015), Estados Unidos (Bassok, Latham \& Rorem, 2016), y también países latinoamericanos como Colombia (Buitrago, 2015), Perú (Ochoa, 2015) y Chile.

En este último país la educación parvularia recae bajo la jurisdicción del Sistema Nacional de Aseguramiento de la Calidad. Entre otras materias, el Sistema sujeta a los establecimientos educacionales al cumplimiento de un conjunto de requisitos para impartir el nivel —e.g., normas de infraestructura- (Ministerio de Educación, 2015) y, en particular para las escuelas — donde está inserta la mayor parte del Nivel Transición ${ }^{8}$ - a evaluaciones nacionales del aprendizaje, una de las cuales es el SIMCE' (Agencia de Calidad de la Educación, 2021). En este marco institucional, la Subsecretaría de Educación Parvularia ha planteado su preocupación por la hiper-escolarización (Ministerio de Educación, 2016, p. 19) del nivel, buscando revertirla mediante orientaciones para elaborar estrategias pedagógicas apropiadas para las transiciones educativas entre educación parvularia y básica (Ministerio de Educación, 2004, 2017). Varias investigaciones han aportado evidencia que respalda la existencia de este fenómeno en el país, derivada de percepciones de educadoras de párvulos y de observaciones en aula. Santibáñez et al. (2009) reportaron que las educadoras de párvulos observaban una fuerte tendencia escolarizante en este nivel educativo, expresada en el plano de los contenidos y, especialmente, en los aspectos metodológicos y disciplinarios. Pardo \& Woodrow (2014) encontraron que las educadoras de párvulos percibían que la escolarización de la educación parvularia está en tensión con los principios pedagógicos del nivel. Pardo \& Opazo (2019) hallaron

8 Según datos del Ministerio de Educación, el 67\% de los niños que asisten al Nivel Transición están matriculados en escuelas (MINEDUC, 2019).

9 Los resultados que obtienen las escuelas en el SIMCE (Sistema de Medición de la Calidad de la Educación) tienen importantes consecuencias sobre los establecimientos. Entre estas se encuentran el riesgo de cierre tras cuatro años consecutivos con desempeño insuficiente y la asignación de incentivos económicos como reconocimiento al buen desempeño (Chile, 2011). La intensidad de las consecuencias asociadas al SIMCE ha sido fuertemente cuestionada, por la presión que implican sobre las comunidades educativas (Campos-Martinez, Corbalan \& Inzunza, 2015) y por perjudicar a los estudiantes más desaventajados socioeconómicamente y con mayores dificultades académicas (Flórez Petour, 2015). 
que las educadoras de párvulos observaban que la pedagogía centrada en el niño estaba siendo amenazada por la escolarización de la educación parvularia. Grau et al. (2018) mostraron que las educadoras de párvulos del Primer Nivel de Transición reconocían encontrarse en un contexto "sobreescolarizado", derivado tanto de las orientaciones de los propios establecimientos, de las expectativas de directoras y familias, como de las políticas públicas para este nivel (e.g., envío de libros más que de materiales lúdicos). Esta misma investigación encontró que, si bien las educadoras declaraban preferir el juego como vehículo del aprendizaje, en la práctica privilegiaban actividades instruccionales; ello en concordancia con los hallazgos de Strasser et al. (2009), quienes encontraron que en aulas del Segundo Nivel de Transición no se dedicaba en absoluto tiempo al juego.

La escolarización de la educación parvularia ha sido interpretada como una tendencia problemática en varios aspectos. En primer lugar, porque restringe las oportunidades de desarrollo integral de los niños y menoscaba así su bienestar, al desatender áreas como su desarrollo socioemocional o motriz (Moss, 2012; OMEP, 2010). En segundo lugar, porque relega a segundo plano estrategias de aprendizaje consideradas naturales y efectivas en los niños pequeños (i.e., juego, exploración, libertad de movimiento, interacciones con otros niños) (Broström, 2006; Hjort, 2006). Y, en tercer término, porque resta centralidad a la dimensión de "cuidado", la cual, así como la dimensión educativa, es parte fundamental del proceso de desarrollo y aprendizaje en la primera infancia (Van Laere, Peeters \& Vandenbroeck, 2012).

La escolarización de la educación parvularia ha implicado un quiebre con las tradiciones pedagógicas más importantes de este campo, como Montessori y Reggio Emilia (OECD, 2004) y la denominada "pedagogía social", que predomina en los países nórdicos y de Europa central. En estos modelos, la educación parvularia representa un nivel educativo en su propio mérito - $-\mathrm{y}$ no una mera preparación para la educación básica-, en el que el aprendizaje es entendido desde una perspectiva holística, el juego cobra relevancia central y el cuidado es inseparable del proceso 
148 ESCOLARIZACIÓN DE LA EDUCACIÓN PARVULARIA EN CHILE: CONSENSOS ENTRE ACTORES DEL CAMPO SOBRE SU DEFINICIÓN, CAUSAS Y PROPOSICIONES - M. Pardo, M. Opazo, P. Rupin

educativo (OECD, 2006, pp. 59-60). Es en esta línea, por cierto, que las Bases Curriculares de la Educación Parvularia (BCEP) de nuestro país anclan sus siete principios pedagógicos, a saber: actividad, singularidad, relación, unidad, juego, significado y potenciación (Ministerio de Educación, 2018).

Para comprender cabalmente la escolarización de la educación parvularia es necesario no abstraer este fenómeno de otros debates que cruzan a este nivel educacional. En primer lugar, la discusión acerca de la relación entre la calidad y los llamados "factores estructurales y de proceso". Los primeros se refieren a los aspectos organizacionales y físicos de las aulas que usualmente están normados: el tamaño del grupo, el curriculum, los recursos para el aprendizaje y el espacio físico. Los factores de proceso se relacionan con las experiencias que involucran a los niños, incluyendo sus interacciones con sus pares, las educadoras y su entorno (Melhuish et al., 2015; Ishmine \& Tyler, 2014).

Un segundo debate concierne a la relevancia del juego en educación parvularia. Sin una definición universal (Pellegrini, 2009, 2013), el juego constituye un derecho de los niños (Naciones Unidas, 1989), a la vez que un medio crítico para su desarrollo físico, intelectual y social (Tremblay et al., 2013). Ocupando un lugar medular desde los albores de la educación parvularia hasta la actualidad (David, Goouch \& Powell, 2015), como puede verse en las BCEP (Ministerio de Educación, 2018), el juego se ha expresado en una variedad de prácticas dentro de este nivel educacional, conformando una diversidad de "culturas preescolares" (Rayna \& Brougère, 2010). Sin perjuicio de ello, existe debate acerca de cómo el juego debiera insertarse dentro de los sistemas educativos y en las prácticas pedagógicas en educación parvularia (Pramling Samuelsson \& Pramling, 2013; Bodrova \& Leong, 2010; Sarlé, 2008; Whitebread et al. 2015), a la vez que cuestionamientos sobre su impacto en tanto actividad pedagógica (Brougère, 2005, 2010).

El tercer debate se refiere al predominio y aplicación de la "forma" educativa escolar sobre la educación parvularia, expresada en el énfasis en normas de comportamiento y en la instalación de 
un formato espacio/temporal caracterizado por la restricción de movimiento, el disciplinamiento del cuerpo, la clara delimitación de tiempos y diferenciación de horarios, y la insistencia sobre la comprensión y seguimiento de consignas e instrucciones (Garnier et al., 2017). Así, también, esta forma educativa escolar ha sido caracterizada por la constitución de un universo que asigna importancia primordial a las reglas en el aprendizaje, la organización racional del tiempo y la multiplicación y repetición de ejercicios (Vincent et al., 1994). Ya tradicional en las sociedades occidentales contemporáneas (Vincent, 1980), esta forma escolar es jerárquica, estructurada y disciplinadora, y se centra en la transmisión de contenidos por la vía de la ejercitación disciplinada, sobre la base de procesos rutinarios y predecibles (Sandoval-Obando y López de Maturana, 2017).

Por último, el cuarto debate es el relativo a la rendición de cuentas en educación parvularia. Este ha sido descrito principalmente en Estados Unidos y el Reino Unido, donde se han instalado sistemas de evaluación de habilidades de los niños — casi siempre, exclusivamente enfocados en lenguaje y matemáticasbuscando determinar si están preparados o no para ingresar a la educación básica (Pianta, 2007; Roberts-Holmes, 2015). Por ejemplo, en Estados Unidos, los resultados obtenidos por el programa Head Start en un conjunto de pruebas estandarizadas se ha vinculado a recortes al presupuesto federal asignado a esta institución (Strauss, 2016).

Pese a la amplia preocupación por esta tendencia, la investigación al respecto es aún escasa, permitiendo una comprensión incipiente sobre la escolarización de la educación parvularia. En particular, la literatura nacional e internacional han indagado escasamente las apreciaciones subjetivas de los actores del campo sobre este fenómeno, dejando en la opacidad sus perspectivas. A través de esta investigación, buscamos contribuir a llenar este último vacío, explorando la visión de distintos actores en el país, examinando cómo educadoras de párvulos, directoras de establecimientos y autoridades nacionales definen la escolarización de este nivel educacional, los 
150 ESCOLARIZACIÓN DE LA EDUCACIÓN PARVULARIA EN CHILE: CONSENSOS ENTRE ACTORES DEL CAMPO SOBRE SU DEFINICIÓN, CAUSAS Y PROPOSICIONES - M. Pardo, M. Opazo, P. Rupin

factores que perciben como sus causas y las proposiciones que plantean al respecto.

\section{Metodología}

\section{Diseño}

Esta investigación se basó en un diseño cualitativo de nivel exploratorio (Jupp, 2006, pp. 110-111).

\section{Recolección de datos}

Los datos fueron recolectados mediante entrevistas semiestructuradas individuales (Corbetta, 2003), basadas en pautas construidas desde la perspectiva etic (Given, 2008). Estas pautas indagaron, de forma específica para cada grupo de participantes, en la definición, causas y proposiciones para la escolarización de la educación parvularia como categorías apriorísticas. Los temas centrales abordados fueron la cobertura de los distintos núcleos de las BCEP, los métodos pedagógicos utilizados, el lugar del juego en las experiencias de aprendizaje, el tipo de disciplina promovido y la preparación de los niños para la escuela. Debido al contexto de pandemia por covid-19 en que se desarrolló esta fase de la investigación, realizamos estas entrevistas vía remota (por Zoom o Google Meet).

\section{Participantes}

Dentro de 10 establecimientos educacionales financiados con recursos públicos que imparten Nivel Medio ${ }^{10}$ y/o Nivel Transición (cinco jardines infantiles y cinco escuelas) en la Región Metropolitana, seleccionados por conveniencia (Jupp, 2006, pp. 196-197), participaron en la investigación tres grupos de actores ${ }^{11}$ : los miembros del equipo directivo de cada establecimiento, es decir, la directora y la jefa de la Unidad Técnico Pedagógica (UTP) de la escuela, y la

10 Nivel Medio es la denominación oficial en establecimientos educacionales para los grupos de niños de dos y tres años. Mayoritariamente, este nivel se imparte en jardines infantiles.

11 Este trabajo adhiere a la perspectiva de género; sin embargo, con el solo propósito de facilitar la lectura, utiliza el género femenino para referirse a educadores y educadoras de párvulos, así como a directores y directoras de establecimientos. 
directora del Jardín Infantil (n=15); la(s) educadora(s) de párvulos (n= 20); y autoridades y exautoridades de instituciones gubernamentales nacionales con jurisdicción sobre el nivel de educación parvularia ${ }^{12}$ $(n=8)$.

Análisis de datos

Empleamos análisis de contenido sobre la base de categorías apriorísticas y emergentes (Andren et al., 2011). Triangulando los datos por actor y tipo de establecimiento (Denzin, 2012), buscamos identificar los principales consensos entre los tres grupos de participantes. Operacionalizamos la noción de "consenso" como la presencia de una categoría analítica (i.e., código) transversal en los tres grupos de participantes, siendo mencionada por al menos la mitad de los participantes de cada grupo. Esto implica que otras categorías de alta densidad, en uno o dos grupos de actores, no fueron seleccionadas para esta publicación.

\section{Aspectos éticos}

Cada participante recibió un documento de consentimiento informado aprobado por el Comité de Ética de la Investigación en Ciencias Sociales y Humanidades de la Facultad de Filosofía y Humanidades de la Universidad de Chile. Este explica resumidamente el proyecto y explicita los derechos de anonimato y confidencialidad de las participantes, así como también su derecho a abandonar la investigación en cualquier momento, sin perjuicio alguno.

\section{Resultados}

La escolarización de la educación parvularia es un fenómeno respecto del que la totalidad de las participantes en esta investigación manifestó tener conocimiento, a partir de sus respectivas experiencias. Los consensos entre los tres grupos de actores participantes emergieron respecto de definiciones, causas y proposiciones de la

12 Este grupo estuvo conformado por altos personeros de distintas agencias del Sistema Nacional de Aseguramiento de la Calidad de la Educación, con directa vinculación con el nivel de educación parvularia, y de instituciones proveedoras de educación parvularia. 
152 ESCOLARIZACIÓN DE LA EDUCACIÓN PARVULARIA EN CHILE: CONSENSOS ENTRE ACTORES DEL CAMPO SOBRE SU DEFINICIÓN, CAUSAS Y PROPOSICIONES - M. Pardo, M. Opazo, P. Rupin

escolarización de la educación parvularia, aunque cada participante no necesariamente estableció un correlato directo entre esas tres dimensiones, sino a veces desarticuladamente. Como evidencia de este consenso, cada uno se presenta a continuación, acompañado de citas textuales para cada actor ${ }^{13}$.

\section{Definiciones}

La escolarización de la educación parvularia fue consensualmente definida desde una perspectiva pedagógica, entendiéndola como un fenómeno relativo a los contextos de aprendizaje — siguiendo la nomenclatura de las BCEP- de los niveles de transición 1 y 2 (con muy menor presencia en el Nivel Medio), caracterizada por la adopción inapropiada de objetivos de aprendizaje y/o métodos propios de la educación básica. En cuanto a los contenidos, los tres grupos de participantes destacaron principalmente el excesivo énfasis en la lectura y, en muy menor medida, en la escritura. A manera de ejemplo se encuentra la pretensión de que los niños desarrollen la conciencia fonológica y aprendan las vocales, y se inicien en la lectura en el Nivel Transición, y, más específicamente, que egresen de Segundo Nivel Transición leyendo.

El énfasis que hemos puesto, así como más grande, es lenguaje. Hay muchos que llegan con problemas de dicción en lenguaje, entonces potenciamos mucho el lenguaje, además... bueno, el lenguaje está en todos lados, pero aún así tratamos de que el lenguaje sea un poco más primordial, porque ellos van a tener que comunicarse más adelante, y ellos son el nivel que va a pasar al colegio. (Educadora 1, Jardín Infantil 3)

Yo tengo experiencias, por ejemplo en colegios particular subvencionados, que se exige la escolarización de los niños, y los niños están estresados aprendiendo a leer en kinder, porque el que no aprende a leer se le sugiere a la mamá que lo deje en el kinder, porque el niño en primero básico ya tiene que venir con la lectura fluida. (UTP, Escuela 5)

13 A fin de maximizar el anonimato de las participantes, en esta sección denominamos "autoridades" tanto a aquellas en ejercicio al momento de la recolección de datos, como a quienes ocuparon este rol anteriormente. 
Los jefes técnicos son los que exigen que el niño tiene que aprender a leer, que tiene que saberse las vocales, etcétera [...] Hay una creencia de ellos que los niños tienen que estar sentados, que tienen que tener... aprender a leer, aprender a escribir, que si no, están perdiendo el tiempo. (Autoridad 8)

Como ejemplo del énfasis en la escritura, se encuentra el objetivo de que los niños aprendan a escribir en el Nivel Transición.

... lo que más me preocupa es que esta nueva dinámica ha ganado terreno durante los últimos años, y que ya son muchas las educadoras que estamos asumidas ya, como que ya es una exigencia que los niños al terminar el kinder deben salir leyendo y con una... no con una excelente caligrafía, pero que sepan escribir, porque eso es lo primero que le piden en primero básico, que casi escriban todo. (Educadora 1, Escuela 3)

De hecho, vienen preparadas [fichas de trabajo]... ellos [una ATE] las mandaban semanalmente, y esa misma lectura era para toda la semana, entonces algunos días se trabajaba en, no sé, de... tratar de escribir el nombre, tratar de escribir las letras iniciales, intermedias, la separación de palabras, entonces todos los días se iban trabajando distintas estrategias que ellas estaban dando desde ahí. (Directora, Escuela 2)

...me voy a enmarcar un poco en la institución escuela [...]. Efectivamente, el deseo o, en el fondo por... el hito cuando tu hijo o hija aprende a leer, aprende a escribir su nombre, el nombre de su familia, empieza a hacer cartas, es una apertura de puertas, en el fondo es un hito en la vida familiar [...]. Y yo creo que es algo muy deseado, en el fondo, que suceda, entonces, efectivamente, puede ser que muchas familias tengan... pongan ese deseo como prioritario. (Autoridad 3)

Las participantes observaron que, como derivación de dicho énfasis en la lectura y la escritura, resulta imposible para las educadoras de párvulos, en términos prácticos, dar cobertura al conjunto de las BCEP. De esta manera, señalaron que el Ámbito de Desarrollo Personal y Social y que el Núcleo Lenguajes Artísticos son los más relegados dentro del curriculum nacional. 
154 ESCOLARIZACIÓN DE LA EDUCACIÓN PARVULARIA EN CHILE: CONSENSOS ENTRE ACTORES DEL CAMPO SOBRE SU DEFINICIÓN, CAUSAS Y PROPOSICIONES - M. Pardo, M. Opazo, P. Rupin

... la interiorización ahora de la escolarización en educación parvularia ha desplazado ese tipo de objetivos de aprendizaje, los cuales siempre fueron trascendentales. El objetivo... todo el Núcleo de Lenguajes Artísticos, al cual no se le da el énfasis que se merece, no se le da el peso que debiese tener, así como tal cual, matemáticas y lenguaje, porque así incluso se plantean en educación parvularia, ya no se plantean como comunicación, sino que se plantea directamente como lenguaje. (Educadora 1, Escuela 2)

Sí, totalmente [abordan todos los ámbitos y núcleos de las BCEP]. Siempre estamos abordando todos los núcleos: el de ciencia, todo; el entorno; la ciudadanía. Todos los núcleos están totalmente abordados. Solamente, les damos más énfasis a esos, pensando que en los colegios le dan más énfasis al lógico-matemático y a la lecto-escritura. (Directora, Jardín Infantil 3)

...porque el sistema educativo, en modo general, está en una competencia tremenda [...], marca un énfasis en objetivos de matemáticas, de lectura, de escritura, como si eso fuera lo único importante. Y lo demás viene a ser un poco pérdida de tiempo, las artes, las ciencias sociales, las ciencias naturales, la transversalidad, el tema, por ejemplo, ahora valórico, los grandes temas, ciudadanía, todo eso como que no lo encuentran valioso. (Autoridad 5)

En cuanto a los métodos, los tres grupos de participantes destacaron que la escolarización implica metodologías pedagógicas inapropiadas en educación parvularia. Así, la lectura es promovida a través de métodos instruccionales, como el uso preferente de lápiz y papel, guías y ejercicios de apresto y grafomotricidad, los que en algunas ocasiones se enmarcaban dentro de programas de fomento lector que las participantes consideraron escolarizados.

.. la guía yo siempre la he encontrado que es una señal de escolarización más que... por ejemplo la rutina, también es una señal de escolarización, como establecer los tiempos súper justos. Por ejemplo, tienes 30 minutos para desarrollar una actividad, o sea que el niño no tiene más tiempo para poder pensar, ¿y si el niño se demora más?, ¿si sus capacidades son distintas?, ¿si su madurez es distinta? No, tienes los 30 minutos, y ahí cortaste, y chao. (Educadora 4, Escuela 3) 
Por ejemplo, el uso de plantillas para poder generar habilidad motriz fina, la fuerza. Yo, el concepto que al menos que yo tengo de niño, es libertad, es autonomía y libertad, entonces un niño que está sentado, no sé, 40 minutos en una silla, con una plantilla y un lápiz, y haciendo en colectivo con todos sus compañeros la misma ficha, eso para mí es escolarizar. Educar es guiar, es ir mediando, motivando a este niño para que él vea hasta dónde pueda llegar y que aprenda... o aprender la misma motricidad fina, pero desde el interés de él, o sea lo centramos en el niño más que en el objetivo de expectativa de aprendizaje. (Directora, Jardín Infantil 5)

...cuando tú entras a una escuela, a un colegio, desgraciadamente a veces incluso lo ves en Nivel Medio [...] un régimen bastante escolarizado tradicional [...] niños muy sentados, haciendo mucha actividad de lápiz y papel, con poco equilibrio a otro tipo de actividades, y con esto, con una extensión, como digo, al ambiente mismo, al ambiente físico, no es habitual el de un jardín infantil, muchas veces con rincones, zonas, materiales a elección, sino tú miras y hay estantes llenos de libros y cuadernos por todos lados, y todos son el libro de matemática, el libro de prelectura, el libro de preescritura, etcétera. (Autoridad 5)

Mención aparte merecen los métodos evaluativos, en tanto metodología pedagógica inapropiada, pues fueron frecuentemente referidos como un ejemplo por antonomasia de la escolarización de la educación parvularia, destacando la aplicación de pruebas y disertaciones, y la calificación mediante notas.

...cuando dicen "Mira, no es una evaluación, los vamos a tomar como un test para saber cómo salen los niños de prekinder", imagínate qué frustrante, qué desgastante para los chiquillos que una persona extraña venga, que tome algo, que es donde tienen que leer, donde tienen que escribir. (Educadora 3, Escuela 3)

...en estos momentos [la pandemia] se ha dado la flexibilidad para que los niños tengan evaluaciones formativas. Y, cuando ellos se reincorporen, ellos van a poder dar nota; van a poder tener las notas y las calificaciones. Pero, creo que en el proceso enseñanza-aprendizaje no todo es nota, no todo es la calificación; y menos para un niño en sus 
156 ESCOLARIZACIÓN DE LA EDUCACIÓN PARVULARIA EN CHILE: CONSENSOS ENTRE ACTORES DEL CAMPO SOBRE SU DEFINICIÓN, CAUSAS Y PROPOSICIONES - M. Pardo, M. Opazo, P. Rupin

primeros años, que además no le va a asignar significado, no entiende lo que le están significando. Además, puede afectar en su autoestima. (Directora, Jardín Infantil 1)

...entonces la educadora dice "bueno ¿qué quiere? Y el director le dice que quiere que le ponga nota a los niños, que ojalá salgan ya leyendo y escribiendo, aunque los tenga toda la mañana en eso. (Autoridad 5)

Una segunda dimensión consensual de la definición de escolarización de la educación parvularia es la cultura escolar que adquiere este nivel educativo, expresada en la construcción de ambientes de aprendizaje caracterizados por la fuerte priorización del aula como el espacio en el que transcurre la jornada, con la consecuente reducción del uso del patio y la biblioteca, la limitación de actividades de educación física, la práctica supresión de salidas pedagógicas fuera del establecimiento y un énfasis en la disciplina estricta de los niños. Otra expresión del predominio de la cultura escolar en los ambientes de aprendizaje de educación parvularia es la fuerte reducción de las oportunidades de juego, tanto dentro como fuera del aula, ejemplificada en la ausencia de rincones de juego y en la breve duración de los tiempos de recreo.

...nos ponían tiempos para trabajar, de tal fecha a tal fecha teníamos que tener trabajados tales contenidos del libro, debiamos avanzar en los libros del colegio, entonces, finalmente..., las actividades de la sala eran actividades de libro, estar sentados, estar enseñándole a los niños "ya, ahora tienen que picar y rellenar", el niño se paraba después de su sala hacia el escritorio del profesor a mostrar su tarea. (Educadora 1, Jardín Infantil 1)

...el colegio te lleva, en cierta medida, a una militarización de los actos. Es decir, a las ocho y treinta acto a la bandera, al aula, desayuno, clases de lenguaje, quince minutos de recreo, y así vamos. En cambio, muchas veces, nosotros en el jardín infantil, si el chico no está bien, salimos a dar una vuelta con él, vamos al huerto; usamos otros aprendizajes: plantamos una semillita, vamos a sacar basura. Aceptamos otras cosas que tienen que ver con todos estos aprendizajes y en la escuela no se permite. (Directora, Jardín Infantil 2) 
...el juego no está prevalecido como una ocupación, una actividad natural del niño, la cual puedes conducir a muchos aprendizajes y un desarrollo integral, entonces, efectivamente, cuando uno puede presenciar una jornada, una mañana, en que hay pocas instancias tanto de juego libre como de juego conducido, yo creo que ambos pueden, no solamente del juego libre, el juego conducido con reglas también puede proporcionar mucho. (Autoridad 3)

La casi totalidad de las participantes en esta investigación (con la excepción de cinco de ellas) asignó una valoración negativa a la escolarización de la educación parvularia, argumentando que esta tendencia tiene un impacto profundamente negativo sobre los niños. En particular, mencionaron un menoscabo al bienestar integral de éstos, señalando como ejemplos su aburrimiento y estrés.

...yo creo que los limita demasiado, los pone nerviosos, como veíamos la evaluación auténtica, el niño no se tiene que sentir calificado, no tiene que sentir ese nerviosismo, algo malo, y eso sí, ellos se sienten presionados. (Educadora 1, Jardín Infantil 5)

A un niño de cinco años no se le puede forzar a pruebas, pruebas esquematizadas, estandarizadas, que los estresan. Tiene que ser un conocimiento y una adquisición de conocimiento de forma entretenida para el niño, que no le quite la etapa de infancia, porque hay ciertos lugares en que eso pasa y el niño, al llegar a básica o al llegar a octavo básico inclusive, poco menos se quiere suicidar porque ya está harto de esa educación tan rígida. (UTP, Escuela 4)

...entender que todo esto no era solamente ese tipo de actividades, sino que habia que desarrollar al niño en ciertas habilidades y en ciertas capacidades que eran más importantes, porque si esas se establecian bien, este niño iba a tener una formación integral más completa, y todos estos aprendizajes academicistas [...] se iban a desarrollar de mucho mejor forma, sin el gran riesgo de niños aburridos, niños que terminan odiando la escuela después, como ha sucedido muchas veces. (Autoridad 5) 
158 ESCOLARIZACIÓN DE LA EDUCACIÓN PARVULARIA EN CHILE: CONSENSOS ENTRE ACTORES DEL CAMPO SOBRE SU DEFINICIÓN, CAUSAS Y PROPOSICIONES - M. Pardo, M. Opazo, P. Rupin

\section{Causas}

En la visión consensual de los tres grupos de participantes, la escolarización de la educación parvularia encuentra tres causas principales: factores estructurales, las aspiraciones y expectativas de las familias, y la incomprensión amplia de los principios pedagógicos de la educación parvularia.

Como ejemplos de factores estructurales, los tres grupos de participantes aludieron a la infraestructura del establecimiento (e.g., existencia de patio techado), el curriculum nacional (i.e., las BCEP), el coeficiente técnico (i.e., número de niños por adulto en el aula) y el tipo de recursos para el aprendizaje disponibles (e.g., libros, material didáctico).

[los niños no podían salir al patio] sobre todo cuando llovía, porque nuestro patio no tiene techo, entonces yo adecuaba la sala con los niños en conjunto, así los niños corrían las mesas, las sillas, y ahí hacíamos juego de roles, yo tenía una maleta grande llena de disfraces, entonces ahi en el mismo momento íbamos "craneando" algo, los dejaba, obviamente, libres. (Educadora 2, Escuela 1)

Yo creo que, mira, las Bases [Curriculares] de la Educación Parvularia no son muy aterrizadas, como que todo es muy vago, entonces a veces en los establecimientos que tienen jornada escolar completa intentan trabajar con esas Bases y son muy poco flexibles, entonces creo que el hecho que tengan, por ejemplo, una jornada extensa, que tengan tantos bloques... ahora, igual uno puede adecuarlo, sobre todo con esas horas que uno tiene de libre disposición, pero yo creo que tiene que ver con la forma en que se presenta el curriculum en la prebásica. (Directora, Escuela 3).

... tenemos un problema de coeficiente técnico súper importante, o sea como de estructura. O sea, pensar que un prekinder y un kinder en Chile legalmente pueden tener 45 niños, es una locura, pensar que pueden jugar 45 niños en un aula, o sea, a menos que sea un galpón... pero no, tampoco, pero en verdad no, el ruido... entonces, ponte tú, yo creo que ahi hay un tema que es importante y que en general siento que 
se tiende a ver que es como si fuera algo menor, si no fuera importante. (Autoridad 2).

Otro factor consensualmente mencionado por los tres grupos de participantes fue la influencia de la forma escolar sobre la educación parvularia. Como un ejemplo frecuentemente mencionado se encuentra la presión que reciben las escuelas, de parte de sus respectivos sostenedores y del Sistema de Aseguramiento de la Calidad de la Educación, para fomentar, desde el Nivel Transición, habilidades que posteriormente permitirán a los niños obtener altos puntajes en el SIMCE.

Yo pienso que el SIMCE ha influido más que nada en eso [la escolarización de la educación parvularia], porque todos quieren puntaje, entonces están exigiendo que el niño, desde muy chico, se meta en un sistema demasiado aguerrido, digamos, o sea, obligándolo a aprender; de hecho, los colegios te vomitan a los niños que no aprenden, cosa que no debiera ser. (Educadora 1, Escuela 4)

Yo creo que las categorías de desempeño que instaló el Sistema de Aseguramiento de la Calidad influyen mucho, porque la gente asume que solo el SIMCE es lo que te ayuda a la categoría, pero están los cuestionarios, están los resultados de eficiencia interna, entre otros. Pero para los apoderados y para las comunidades educativas, ser academicistas en sus proyectos educativos es señal de buena calidad de la educación; entonces, yo creo que a eso, a eso se atribuye, es sentir que, en realidad, mientras más exigente, mientras más atosiguemos un poco a los niños, porque tan chiquititos y están súper estresados, es señal de que es un colegio bueno. (Directora, Escuela 3)

Aunque en las Bases Curriculares se resguardó que no se propicie la enseñanza de la lectura formal, sino más bien las habilidades de prelectura, si pasa mucho que se presiona. ¿Por qué?, porque una vez que ya manejas el código, entonces la vida cambia... la vida cambia para los profesores también, entonces yo creo que ahi hay un tema, una presión del sistema que se podría asociar efectivamente a tener un mejor resultado en SIMCE. (Autoridad 1) 
160 ESCOLARIZACIÓN DE LA EDUCACIÓN PARVULARIA EN CHILE: CONSENSOS ENTRE ACTORES DEL CAMPO SOBRE SU DEFINICIÓN, CAUSAS Y PROPOSICIONES - M. Pardo, M. Opazo, P. Rupin

Un segundo ejemplo de la influencia de la forma escolar sobre la educación parvularia fueron las aspiraciones y expectativas de parte de las familias respecto de los niños. Los tres grupos de participantes plantearon que éstas se traducen en una presión sobre los establecimientos para que centren su trabajo pedagógico en lenguaje y matemáticas, con el fin de asegurar que los niños egresen del Nivel Transición ya leyendo, y de prepararlos para rendir un buen examen de admisión en las escuelas a las que postulan.

...una de las preguntas [que el jardín plantea a los apoderados a través de un cuestionario] dice "iqué espera usted del equipo educativo?". Y muchas veces nos pasa que... bueno, después nosotros vamos a leer esas respuestas, porque tenemos que tabular y todo: "que aprendan los colores, que aprendan los números"; incluso me ha pasado que hay apoderados que dicen "los niños no han aprendido nada porque no saben contar”. (Educadora 2, Jardín Infantil 5)

...muchos apoderados nos dicen "mi hijo no aprendió nada, nada en kinder", porque cree que no aprende nada, y nosotros le explicamos, igual que las tareas: "profesora, no nos dan nada de tarea", y la profesora hace un montón de trabajos lúdicos, les hace juegos de mesa, aqui por ejemplo todavía quedan algunos juegos de mesa que las profesoras les han hecho a los niños, entonces ellos sienten, ellos creen que eso no es aprender. (UTP, Escuela 5)

...creo que incluso también hay un tema como social, como de que los papás, cuando llevan a sus hijos al colegio versus al jardín, están esperando que hagan tareas [...] como una visión escolarizada de los papás de la escuela [...] que van y dicen " ¿y dónde están los trabajos de mis hijos?" [...]. Creo que hay un tema social también, que influye, que tiene que ver con que la adquisición de cierto contenido se asocia como al éxito, como a las posibilidades de éxito del niño, entonces el papá lo que más quiere es que el niño aprenda los números y las letras lo antes posible, entonces eso juega en contra. (Autoridad 2).

La tercera causa de la escolarización es la amplia incomprensión sobre principios pedagógicos de la educación parvularia. En particular, los tres grupos de participantes señalaron un desconocimiento sobre 
las metodologías que son pertinentes a este nivel, poniendo especial relieve en la incomprensión del rol del juego en la educación de la primera infancia, llegando a desestimarlo en su relevancia.

Yo creo, sinceramente, que las personas que hacen esos protocolos como que están enfocados en el departamento de educación, yo creo que nunca han estado en una sala de clases, en una sala de clases de un kinder, de un prekinder, de una sala cuna, de los niveles medios, nunca han estado ahí, y lo que ven por fuera, como le decía yo, que decían "no, si en kínder van a pintar, a jugar, esto... los números aquí, van a puro...". (Educadora 2, Escuela 2)

...en la escuela en que trabajé todavía trabaja mi amiga, entonces me dice "oye, ustedes, los niños son un desastre, no saben hacer nada, solo quieren jugar, y nosotros no necesitamos eso". Entonces ahi empieza un poco la pugna de la idea, de la concepción que tenemos como jardines, de lo que debe ser la educación parvularia versus la idea que tiene preconcebida ella de lo que tiene que entregar como niño para primero básico. (Directora, Jardín Infantil 5)

...cómo transmitirles que también, por ejemplo, que los niños que tienen más creatividad y aprenden, y juegan más, y todo eso, después van a tener mejores resultados [...], que no es que el niño va a chuparse el dedo, sino que el niño aprende a hacer experimentos, aprende a hacerse preguntas, a hacer hipótesis, a ver cómo lo resuelve, y que con eso le va a ir mejor. Eso es súper difícil de transmitir, yo creo que por ahi tiene que ser la apuesta, que vea que lo que está haciendo sí es que los niños adquieran mejores, o sea mayores habilidades, pero de acuerdo a sus capacidades y a como a la modalidad que va a aprender más. (Autoridad 2).

\section{Proposiciones}

Tres proposiciones, todas dirigidas al ámbito de las políticas públicas, fueron consensualmente planteadas por los tres grupos de participantes: fortalecer el respeto a los principios pedagógicos de la educación parvularia dentro de los establecimientos educacionales, refinar la articulación curricular con primero básico y difundir los 
162 ESCOLARIZACIÓN DE LA EDUCACIÓN PARVULARIA EN CHILE: CONSENSOS ENTRE ACTORES DEL CAMPO SOBRE SU DEFINICIÓN, CAUSAS Y PROPOSICIONES - M. Pardo, M. Opazo, P. Rupin

principios pedagógicos de la educación parvularia entre los diversos actores de este nivel educativo.

Respecto el fomento de los principios pedagógicos de la educación parvularia al interior de los establecimientos, las entrevistadas señalaron la necesidad de poner de relieve los intereses y elecciones de los niños, el respeto a sus ritmos de aprendizaje y la búsqueda de su desarrollo y bienestar integral. En consecuencia, sugirieron medidas como el realce del juego (e.g. adquiriendo una ludoteca), el uso de métodos evaluativos apropiados (e.g., listas de cotejo, escala de apreciación, rúbricas, observación anecdótica), el uso más intensivo de espacios fuera del aula (e.g., salir más frecuentemente al patio, salir del establecimiento a recorrer el barrio, visitar áreas verdes).

Nosotros los sacamos [a los niños] a la plaza, o dar una vuelta a la manzana, le mostramos los árboles, las hojas, los rayos, que las flores tienen pétalos; o sea, hacemos toda una clase mientras caminamos por algún rincón del barrio. (Educadora 1, Escuela 4)

Porque realizamos una evaluación más cualitativa. Realizamos una evaluación de otra manera. Entonces, claro, yo creo que ya después, en la educación básica, puede que a lo mejor tengan otras presiones, porque tienen que responder a otras cosas también. (Directora, Jardín Infantil 1)

...cuando ese juego tiene intencionalidad y tiene énfasis y tiene una mediación, ese juego va a ser mucho más potente que las guías de grafomotricidad, en que a cada hoja completar con as, bes, ces hacia abajo. O la olita... esa olita se puede hacer, pero de una forma mucho más entrete... también se puede hacer, pero de una forma entretenida, vinculante, y que tenga mucha más trascendencia, en el fondo. Entonces, de la olita después nos pasamos al hacer, lo hicimos primero la olita con nuestro cuerpo, tiramos agua con una manguera, con esta forma, y vimos cómo se fue haciendo la forma y el involucramiento. (Autoridad 3)

La segunda proposición, el refinamiento de la articulación curricular con el primer año básico, fue ilustrada en relación con 
la necesidad de reforzar el trabajo colaborativo entre los equipos pedagógicos de ambos niveles educacionales, y de extender el uso de las metodologías pedagógicas propias de la educación parvularia en los primeros años de educación básica.

...primero [básico] también podría tener un trabajo colaborativo con nosotros, pero adaptarse ellas [las profesoras de $1^{\circ}$ básico] también a la metodología de la escuela, no en infraestructura, porque es bastante dificil tener estas mismas mesas que tenemos nosotros [...]; el tema del juego, de poder tener rincones dentro de tu sala. Poder tener una biblioteca de aula con cojines donde ellos pudieran disfrutar de una lectura, no en su silla, en su mesa, que sea algo más libre, algo que se disfrute, que si se quedó dormido estaba súper relajado. (Educadora 2, Escuela 3)

Creo que el equilibrio debiera ser el punto en que permitamos que los niños se vayan acercando al proceso educativo que van a vivenciar cuando pasen a básica, pero sin perder el foco de que la educación de prebásica, que no es que los niños aprendan a leer, no es que salgan con las habilidades que deben aprender en primero, sino que se acerquen de otra forma a lo que es la educación, que vayan trabajando con sus compañeros, con las educadoras, que vayan experimentando, que vayan conociendo cosas de su entorno, de sus barrios, de nuestra misma escuela, creo que eso no debe perderse. (Directora, Escuela 3).

Yo pienso que ojalá termináramos con la escolarización: o sea, primera infancia hasta los ocho años, y con una metodología de trabajo centrada, por un lado, a esta diversidad ¿no?, con todo lo que el niño trae, con ofrecerles posibilidades a través de descubrimiento, a través del juego, a través de una actividad de hacer aprendiendo, más que sentados con un profesor al frente. (Autoridad 7).

Para la proposición de difundir — tanto entre familias, directoras de establecimientos educacionales, como entre autoridades nacionales- los principios pedagógicos de la educación parvularia, las entrevistadas dieron como ejemplo, en el establecimiento, la realización de charlas a las familias durante las reuniones de apoderados; en el nivel de las políticas públicas, que el Ministerio de 
164 ESCOLARIZACIÓN DE LA EDUCACIÓN PARVULARIA EN CHILE: CONSENSOS ENTRE ACTORES DEL CAMPO SOBRE SU DEFINICIÓN, CAUSAS Y PROPOSICIONES - M. Pardo, M. Opazo, P. Rupin

Educación implemente, en todo el país, campañas comunicacionales y formativas dirigidas tanto a directoras de establecimientos como a las familias, para orientar sobre los objetivos y metodologías propios de este nivel educacional.

...sí, el apoderado te lo exige y bueno, yo llegué en mis últimos años de educadora de escuela a hacer entender a los apoderados que no era necesario, que no era necesario, que habia muchas otras actividades que sí nosotros le podíamos sacar mucho más partido. (Educadora 1 , Jardín Infantil 4)

Es que yo creo que ahí las políticas públicas se tendrían que preocupar de eso [difundir los principios pedagógicos de la educación parvularia], para que los directores y los UTP, en este caso, conocieran un poco más la forma de trabajar de la educación inicial. Yo creo que el Ministerio de Educación es fundamental en este cambio, en esta transición. (Directora, Jardín Infantil 3)

Las primeras reuniones deberían partir con la familia, imaginate, tenemos... toda educadora tiene ahi a la familia en la mano. ¿Y con qué parte la primera reunión? No parte hablando qué es la buena educación parvularia y, coherente con ello, cuál es su proyecto. No, parte mostrando las cosas maravillosas que saben hacer los niños, entonces parte con cosas súper triviales "que ya viene esto, que las peticiones de esto y lo otro". (Autoridad 5)

\section{Discusión}

En una breve recapitulación de nuestros hallazgos, en la visión consensual de las educadoras de párvulos, las directoras de establecimientos educacionales y las autoridades y exautoridades nacionales del nivel que participaron en esta investigación, la escolarización de la educación parvularia fue definida, principalmente, en términos del énfasis en la lectura, el escaso espacio para el juego, el uso de metodologías pedagógicas inapropiadas y el predominio de contextos de aprendizaje — según la nomenclatura de las BCEPpropios de la educación básica. Valorándola negativamente, por implicar un menoscabo al bienestar integral de los niños, estos tres 
grupos identificaron tres causas principales: un conjunto de factores estructurales, el predominio de la forma escolar y la incomprensión de los principios pedagógicos de la educación parvularia. Para superar esta tendencia las participantes propusieron medidas de política pública.

Ratificando lo reportado por investigaciones nacionales anteriores (Grau et al., 2018; Pardo \& Opazo, 2019; Pardo \& Woodrow, 2014; Santibáñez et al., 2009), estos hallazgos encienden alarmas. Por una parte, porque las manifestaciones de la escolarización de la educación parvularia ya descritas refieren a prácticas reconocidas como un menoscabo a las oportunidades de desarrollo integral (e.g., énfasis en la lectura) y de bienestar (e.g., escaso tiempo fuera del aula) (Moss, 2012; OMEP, 2010).

Por otra parte, nuestros hallazgos alarman porque las participantes señalan como causa de la escolarización de la educación parvularia un conjunto de atributos de nuestro sistema educacional, reflotando largos debates sobre nuestro modelo. Así, la percepción de que la escolarización radica en parte en factores estructurales (e.g., falta de patio techado, escasa variedad de recursos para el aprendizaje) como causa de la escolarización, vuelve a señalar la seriedad de este tipo de deficiencias de la calidad de la educación parvularia en Chile, ya detectadas en trabajos anteriores (CEPI, 2017; Rivera et al., 2014).

Así, también, la forma escolar señalada como una causa importante de la escolarización de la educación parvularia, reflejada en expectativas familiares de lectura temprana y en una amplia incomprensión de los principios pedagógicos de este nivel, trae a colación una discusión que, en Chile, no se ha dado con la suficiente detención: la relación entre este nivel y la educación básica. Se trata por cierto de un tema irresuelto en casi todo el mundo (Moss, 2012), pero que, frente a su vínculo con la escolarización, a nuestro país le urge abordar en todas sus ramificaciones institucionales y pedagógicas, incluyendo la influencia del SIMCE, al que las participantes identificaron como un ejemplo notable, pese a que esta medición no se aplica en educación parvularia. 
166 ESCOLARIZACIÓN DE LA EDUCACIÓN PARVULARIA EN CHILE: CONSENSOS ENTRE ACTORES DEL CAMPO SOBRE SU DEFINICIÓN, CAUSAS Y PROPOSICIONES - M. Pardo, M. Opazo, P. Rupin

En su conjunto, dichos hallazgos resitúan el nivel del debate, al apuntar hacia dimensiones del sistema educativo de nivel macro, mucho más allá de las prácticas escolarizadas individuales de educadoras de párvulos y directoras. Concordando con las proposiciones planteadas por las participantes de esta investigación, pensamos que sus resultados respaldan la adopción de medidas más amplias y enérgicas de carácter sistémico. En específico, ante el aparente escaso impacto de las orientaciones sobre articulación y transiciones hacia la educación básica entregadas por el Ministerio de Educación $(2004,2017)$, creemos necesario que el Sistema Nacional de Aseguramiento de la Calidad, en su conjunto, tome acciones expresamente dirigidas a frenar esta negativa tendencia. En primer lugar, reforzando, a través de la Agencia de Calidad de la Educación, el acompañamiento pedagógico a sostenedores, directoras de escuela y educadoras de párvulos, con el fin de asegurar una cobertura balanceada de los Núcleos de las BCEP, ampliar las oportunidades para el juego y emplear los métodos evaluativos apropiados a este nivel. En segundo, cautelando el apego estricto a los Estándares Indicativos de Desempeño para los establecimientos que imparten educación parvularia y sus sostenedores (Ministerio de Educación, 2020), por medio de las visitas de la Agencia, de modo de resguardar prácticas pedagógicas apropiadas a este nivel educacional y, así, frenar la propagación de los efectos perniciosos del SIMCE como mecanismo de rendición de cuentas (Campos-Martinez, Corbalan \& Inzunza, 2015; Flórez Petour, 2015) en este nivel. Y, en tercer lugar, elevando el presupuesto que el Ministerio de Educación asigna para mejorar la infraestructura y el equipamiento de los establecimientos, con el propósito de ampliar y diversificar los espacios educativos y recursos para el aprendizaje disponibles.

En cuanto a la influencia de la forma escolar, se trata de un tema cuya solución no es evidente hoy, dada la escasa comprensión que tenemos al respecto. Para avanzar en esta línea, pensamos que la Subsecretaría de Educación Parvularia debiera superar el enfoque normativo adoptado previamente (Ministerio de Educación, 2004, 2017), liderando una reflexión nacional que abarque a todo el campo en torno a la aparente contradicción entre las expectativas de logro 
académico y los fundamentos pedagógicos de la educación parvularia, buscando vías para conciliarlos.

Pero ello no es suficiente, pues, siendo ésta una primera exploración sobre esta tendencia, también se requiere más investigación para entender mejor aspectos medulares del problema, como la amplitud y la intensidad que adquiere en los distintos tramos de la educación parvularia (i.e., Sala Cuna, Nivel Medio, Nivel Transición) y en las distintas instituciones proveedoras.

\section{Referencias}

Andren, G. et al. (2000). Classical content analysis: a review. In Bauer, M. W., \& Gaskell, G. (Eds.), Qualitative researching with text, image and sound (pp. 132-151). SAGE Publications Ltd, https://www.doi. org/10.4135/9781849209731

Agencia de Calidad de la Educación. (2021). ¿Quiénes somos? Recuperado de: https://www.agenciaeducacion.cl/quienes-somos/

Alcock, S. \& Haggerty, M. (2013). Recent policy developments and the "schoolification" of early childhood care and education in Aotearoa New Zealand. Early Childhood Folio, 17(2), 21-26.

Bassok, D., Latham, S. \& Rorem, A. (2016). Is Kindergarten the New First Grade? AERA Open, 2(1), 2332858415616358. DOI: https://doi. org/10.1177/2332858415616358

Brogaard Clausen, S. (2015). Schoolification or early years democracy? A cross-curricular perspective from Denmark and England. Contemporary Issues in Early Childhood, 16(4), 355-373.

Bodrova, E. \& Leong, D.J. (2010). Curriculum and Play in Early Child Development. In: Tremblay RE, Boivin M, Peters RDeV, eds. Smith PK, topic ed. Encyclopedia on Early Childhood Development [online]. Retrieved from http://www.child-encyclopedia.com/play/accordingexperts/curriculum-and-play-early-child-development. Published September 2010. Accessed September 30, 2020.

Broström, S. (2006). Curriculum in preschool. International Journal of Early Childhood, 38(65).

Brougère, G. (2005). Jouer/Apprendre. Paris: Economica.

Brougère, G. (2010). Formes ludiques et formes éducatives. In J. Bédard \& G. Brougère (Eds.), Jeu et apprentissage, quelles relations? (pp. 43-62). Sherbrooke: Les Éditions du CRP. 
168 ESCOLARIZACIÓN DE LA EDUCACIÓN PARVULARIA EN CHILE: CONSENSOS ENTRE ACTORES DEL CAMPO SOBRE SU DEFINICIÓN, CAUSAS Y PROPOSICIONES - M. Pardo, M. Opazo, P. Rupin

Buitrago, N. (2015). Informe Nacional sobre Docentes para la Educación de la Primera Infancia: Colombia. Retrieved August 26, 2015, from http://www.politicasdocentesalc.cl/images/stories/Biblioteca/ InformePrimeraInfanciaCOLOMBIA WEB.pdf

Cameron, C. \& Moss, P. (Eds.). (2011). Social Pedagogy and Working with Children and Young People: Where Care and Education Meet. Londres, Filadelfia: Jessica Kingsley Publishers.

Campos-Martinez, J., Corbalan, F. \& Inzunza, J. (2015). Mapping neoliberal reform in Chile: Following the development and legitimation of the Chilean system of School quality Measurement (SIMCE). In W. Au \& J. Ferrare (Eds.), Mapping Corporate Education Reform: Power and Policy Networks in the Neoliberal State (pp. 106-125). New York: Routledge Taylor \& Francis.

CEPI. (2017). Calidad en ambientes educativos JUNJI. Recuperado de: https:// www.junji.gob.cl/wp-content/uploads/2019/05/Resumen-FinalEstudio-Calidad-en-Ambientes-Educativos-JUNJI.pdf

Corbetta, P. (2003). The qualitative interview. In Social research: Theory, methods and techniques (pp. 264-286). SAGE Publications, Ltd, DOI: https://www.doi.org/10.4135/9781849209922

David, T., Goouch, K. \& Powell, S. (Eds.). (2015). (Editor). The Routledge International Handbook of Philosophies and Theories of Early Childhood Education and Care. Routledge.

Denzin, N. Triangulation 2.0. Journal of Mixed Methods Research. 2012; 6(2):80-88. doi:10.1177/1558689812437186

Flórez Petour, M. T. (2015). Validity and equity in educational measurement: The case of SIMCE. Psicoperspectivas, 14(3), 31-44.

Garnier, P., Rayna, S., Brougère, G. \& Rupin, P. (2017). Children of two to three years of age in France: early childhood settings and age divisions European Early Childhood Education Research Journal, 25(3), 346-358

Given, L. M. (2008). The SAGE encyclopedia of qualitative research methods (Vols. 1-0). Thousand Oaks, CA: SAGE Publications, Inc. DOI: $10.4135 / 9781412963909$

Grau, V., Preiss, D., Strasser, K., Jadue Roa, D., López, V. y Whitebread, D. (2018). Rol del juego en la educación parvularia: creencias y prácticas de educadoras del nivel de transición menor. Proyecto FONIDE FX11615. Santiago: MINEDUC. Recuperado de: https://centroestudios.mineduc. cl/wp-content/uploads/sites/100/2018/10/Informe-final-FONIDEFX21615-Grau_apDU.pdf 
Gunnarsdottir, B. (2014). From play to school: are core values of ECEC in Iceland being undermined by 'schoolification'? International Journal of Early Years Education, 22(3), 242-250. DOI: https://doi.org/10.1080/ 09669760.2014.960319

Hjort, K. (2006). De-Democratisation in Denmark? European Educational Research Journal, 5(3-4), 234-243. DOI: https://doi.org/10.2304/ eerj.2006.5.3.234

Ishmine, K. \& Tyler, C. (2014). Assessing Quality in Early Childhood Education and Care. European Journal of Education, 49(2). DOI: 10.1111/ejed.12043

Jupp, V. (2006). The SAGE Dictionary of Social Research Methods. London: SAGE Publications.

Melhuish, E., Ereky-Stevens, K., Petrogiannis, K., Ariescu, A., Penderi, E., Rentzou, K., ... Broekhuisen, M. (2015). A review of research on the effects of early childhood education and care (ECEC) on child development. CARE. Curriculum and quality analysis and impact review of European Early Childhood Education and Care. Retrieved from http:// ececcare.org/fileadmin/careproject/Publications/reports/new_version_ CARE_WP4_D4_1_Review_on_the_effects_of_ECEC.pdf

Ministerio de Educación. (2004). Resolución 11636 exenta. Imparte criterios técnicos sobre articulación curricular entre los niveles de educación parvularia y enseñanza básica. Recuperado de: https://www.bcn.cl/ leychile/navegar?idNorma=231212

Ministerio de Educación. (2015). Modifica Decreto Supremo N 315, de 2010, del Ministerio de Educación, que reglamenta requisitos de adquisición, mantención y pérdida del Reconocimiento Oficial del Estado a los establecimientos educacionales de educación parvularia, básica y media. Recuperado de: https://www.bcn.cl/leychile/navegar?idNorma=108 2169\&buscar=decreto\%2B264\%2Bde\%2B2015

Ministerio de Educación. (2016). Hoja de ruta. Definiciones de política para una educación parvularia de calidad. Santiago, Chile: MINEDUC. Recuperado de: https://parvularia.mineduc.cl/hoja-de-ruta/

Ministerio de Educación. (2017). Establece principios y definiciones técnicas para la elaboración de una estrategia de transición educativa para los niveles de educación parvularia y primer año de educación básica. Recuperado de: https://parvularia.mineduc.cl/wp-content/uploads/sites/34/2018/05/ Decreto-Transici\%C3\%B3n-373.pdf

Ministerio de Educación. (2018). Bases Curriculares Educación Parvularia. Santiago, Chile: MINEDUC. 
170 ESCOLARIZACIÓN DE LA EDUCACIÓN PARVULARIA EN CHILE: CONSENSOS ENTRE ACTORES DEL CAMPO SOBRE SU DEFINICIÓN, CAUSAS Y PROPOSICIONES - M. Pardo, M. Opazo, P. Rupin

Ministerio de Educación. (2019). Informe de caracterización de la educación parvularia. Descripción estadística del sistema educativo asociado al nivel de Educación Parvularia en Chile. Santiago, Chile: Ministerio de Educación.

Ministerio de Educación. (2020). Estándares Indicativos de Desempeño para los establecimientos que imparten Educación Parvularia y sus sostenedores. Recuperado de: https://parvularia.mineduc.cl/wp-content/uploads/ sites/34/2020/08/Estandares-Indicativos-EID-EP.pdf

Ministerio de Educación. (2011). Ley 20529 Sistema Nacional de Aseguramiento de la Calidad de la Educación Parvularia, Básica y Media y su Fiscalización. Santiago, Chile: MINEDUC.

Moss, P. (2002). Time to Say Farewell to 'Early Childhood'? Contemporary Issues in Early Childhood, 3(3), 435-438. DOI: https://doi.org/10.2304/ ciec.2002.3.3.9

Moss, P. (2012). The relationship between early childhood and compulsory education: A properly political question. In P. Moss (Ed.), Early Childhood and Compulsory Education: Reconceptualising the relationship (Contesting Early Childhood) (pp. 2-50). Routledge.

Naciones Unidas. (1989). Convención sobre los Derechos del Niño. Recuperado de: https://www.un.org/es/events/childrenday/pdf/derechos.pdf

Ochoa, S. (2015). Informe Nacional sobre Docentes para la Educación de la Primera Infancia: Perú. Recuperado de: http://www.politicasdocentesalc. com/images/stories/Biblioteca/InformePrimeraInfanciaPERÚweb.pdf

OECD. (2004). Five Curriculum Outlines. Paris: Starting Strong Curricula and Pedagogies in Early Childhood Education and Care.

OECD. (2006). Starting strong II: Early childhood education and care. Paris: OECD Publishing.

OECD. (2012). Quality Matters in Early Childhood Education and Care. Retrieved June 7, 2017, from http://www.oecd-ilibrary.org/education/qualitymatters-in-early-childhood-and-education_22269673

OMEP. (2010). World declaration about the right and the joy to learn through play. Retrieved from http://www.cepchileag.cl/index.php?option=com_co ntent\&view=article\&id=189: colegio-de-educadores-de-parvulospublica-declaracion-de-laasamblea-y-el-congreso-mundial-deomep\&catid=40: noticias $\&$ Itemid $=59$

Pardo, M. \& Opazo, M.-J. (2019). Resisting schoolification from the classroom. Exploring the professional identity of early childhood teachers in Chile / Resistiendo la escolarización desde el aula. Explorando la identidad profesional de las docentes de primera infancia en Chile. Cultura y 
Educación, 31(1), 67-92. DOI: https://doi.org/10.1080/11356405.2 018.1559490

Pardo, M. \& Woodrow, C. (2014). Improving the quality of early childhood education in Chile: tensions between public policy and teacher discourses over the schoolarisation of early childhood education. International Journal of Early Childhood, 46(1), 101-115.

Pellegrini, A. (2009). The role of play in human development. New York: Oxford University Press.

Pellegrini, A. (2013). Play. In Zelazo, P. (Ed.). The Oxford Handbook of Developmental Psychology, Vol. 2: Self and Other. DOI: 10.1093/ oxfordhb/9780199958474.013.0012

Pianta, R. (2007). Early education in transition. In R. Pianta, M. Cox \& K. Snow (Eds.), School readiness and the transition to kindergarten in the era of accountability (pp. 3-10). Baltimore, MD: Brooks Publishing.

Pramling Samuelsson, I. \& Pramling, N. (2013). Play and Learning. In: Tremblay RE, Boivin M, Peters RDeV, eds. Smith PK, topic ed. Encyclopedia on Early Childhood Development [online]. Retrieved from: http://www.child-encyclopedia.com/play/according-experts/play-andlearning. Published June 2013. Accessed March 5, 2021.

Rayna, S. \& Brougère, G. (2010). Jeu et cultures préscolaires. Lyon: INRP.

Rivera, L., Faverio, F., Cortázar, A. y Vielma, C. (2014). Hacia una educación parvularia de calidad: ¿Qué costos tendría para Chile?. Informes para la Política Educativa, 4. Recuperado de: https://cpce.udp.cl/wp-content/ uploads/2016/08/ipe4.pdf

Roberts-Holmes, G. (2015). The 'datafication' of early years pedagogy: 'if the teaching is good, the data should be good and if there's bad teaching, there is bad data.' Journal of Education Policy, 30(3), 302-315.

Sandoval-Obando, E. y López de Maturana, S. (2017). Desafíos Educativos en Torno a las Experiencias de Aprendizaje Mediado con Adolescentes Infractores de Ley. Profesorado, Revista del Currículum y Formación del Profesorado, 21(2), 377-391.

Santibáñez, D., Meersohn, C., Lasnibat, M., Torrejón, M. J., Raglianti, F., Egaña, G. y Jorquera, P. (2009). Requerimientos técnicos pedagógicos de las/los educadoras/es de párvulos en el marco de la Ley SEP. Santiago: PULSO, Facultad de Ciencias Sociales, Universidad de Chile.

Sarlé, P. (2008). Enseñar en clave de juego. Enlazando juegos y contenidos. Buenos Aires: Novedades Educativas. 
172 ESCOLARIZACIÓN DE LA EDUCACIÓN PARVULARIA EN CHILE: CONSENSOS ENTRE ACTORES DEL CAMPO SOBRE SU DEFINICIÓN, CAUSAS Y PROPOSICIONES - M. Pardo, M. Opazo, P. Rupin

Strasser, K., Lissi, M. y Silva, M. (2009). Gestión del Tiempo en 12 Salas Chilenas de Kindergarten: Recreo, Colación y Algo de Instrucción. Psykhe (Santiago), (18), 85-96.

Strauss, V. (2016). How a preschool test can endanger Head Start program funding. En https://www.washingtonpost.com/news/answer-sheet/ wp/2016/05/20/how-a-preschool-test-can-endanger-head-startprogram-funding/

Tremblay et al. (2013). Play: Synthesis. In Encyclopedia on Early Childhood Development [online]. Retrieved from http://www.child-encyclopedia. com/play/synthesis. Updated June 2013. Accessed March 5, 2021.

Van Laere, K., Peeters, J. \& Vandenbroeck, M. (2012). The Education and Care Divide: the role of the early childhood workforce in 15 European countries. European Journal of Education, 47(4), 527-541. DOI: https:// doi.org/10.1111/ejed.12006

Vincent, G. (1980). Lécole primaire française : étude sociologique. Lyon : PUL.

Vincent, G., Lahire, B. \& Thin, D. 1994. Sur l'histoire et la théorie de la forme scolaire. In G. Vincent (Ed.), L'Éducation prisonnière de la forme scolaire? Scolarisation et socialisation dans les sociétés industrielles. Presses universitaires de Lyon. DOI: 10.4000/books.pul.9552

Whitebread, D., Kuvalja, M. \& O'Connor, A. (2015). Quality in Early Childhood Education: an International Review and Guide for Policy Makers. Cambridge, UK: University of Cambridge.

Recibido: 14/01/2021

Aceptado: 12/04/2021 